\title{
Degradation Feature Analysis of Escalator Step Chain Based on SIMPACK
}

\author{
Lirui Zhao ${ }^{a}$ and Xiukun Wei ${ }^{b}$ \\ Beijing Jiao tong University, Beijing 100044, China. \\ a16120960@bjtu.edu.cn, b xkwei@bjtu.edu.cn
}

Keywords: SIMPACK, Simulation, Time Domain Analysis, Frequency Domain Analysis.

\begin{abstract}
In this paper, the escalator step chain model is established in SIMPACK a dynamic simulation software to simulate the step chain with different degrees of degradation. And then the data collected in degraded escalator step chain path is analyzed respectively by time domain method and frequency domain method. The analysis results show the reliability of the step chain degradation model based on SIMPACK which provides an important reference for degradation detection of step chain in real life.
\end{abstract}

\section{Introduction}

As one of the most important basic station equipment in the rail transit system, escalators transport thousands of passengers every day, but accidents associated with them are also frequent, causing major casualties and property losses, and related safety issues catch much attention. Step chain is an important part of escalators and it is also a part that is prone to failure and its safety status directly affects the operation of escalators.

Currently, the methods in which escalator step chain is maintained are based on Time Based Maintenance and Run-to-Breakdown Maintenance, and there are few researches on step chain degradation. The most research on degradation law of general chains is mainly based on a large number of experiments, which will take a lot of manpower, material and time. The follows are papers studying on chains. The paper [1] design an experimental device that can test the life of the escalator step roller and predict the life of the roller through a large number of experiments. The paper [2] studied experimentally that the lifetimes of automotive chain under high stress test conditions comply with Weibull distribution with two parameters, the form parameter and the measure parameter which augment with the test load. The paper [3] studied the fractured pin using microscopes and spectrometers, discovering that the main fracture reasons were the grinding stress and quenching retained stress on the axle-pin surface. And with the cooperation of the stress and hydrogen, hydrogen ductile-brittle fracture occurred on the surface. In paper [4], nondestructive testing methods was used to the surface defects of scraper conveyor chain. And the paper measures the elongation of the chain using chain measuring ruler. The paper [5] simulated the change of the longitudinal force of a certain link, the contact force of certain inner and outer link plate, the linear velocity of a certain link during one cycle, which studied by three kinds of dynamic simulation software.

The method proposed in this paper can effectively save time, manpower, material and financial resources, and can also obtain the state parameters of the cascade chain in real time, which provides an important basis for the maintenance and repair of the escalator step chain.

\section{Analysis of Transmission Characteristics of Chain Drive}

\subsection{Polygonal Effect of Chain Drive}

The kinematics characteristic of the chain drive is that the path of the chain around the gear is composed of polygons. This characteristic is the polygon effect of the chain. When the gear rotates, the centerline of the chain will periodically vibrate up and down as the chain meshes with the corresponding gear. 
As shown in Figure 1, the centerline of the chain and the pitch circle with radius $r$ on the sprocket perform tangential and intersecting movements. Due to this polygonal effect of the chain, both the linear velocity of the chain and the angular velocity of the driven wheel change periodically.

(a)

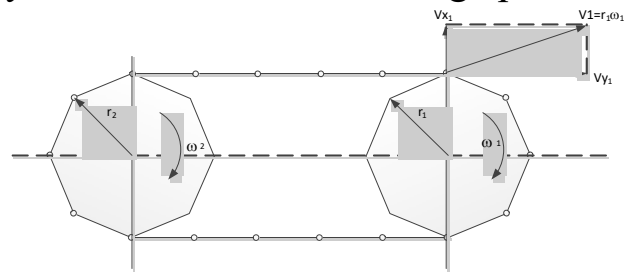

(b)

\subsection{Polygonal Effect of Chain Drive}

Fig 1. The change of chain velocity

When the driving wheel moves at a constant angular velocity, the movement state of the chain in figure 1 is analyzed. Respectively, the velocity of the chain along the center line, that is, the linear velocity $v_{x 1}$ of the chain, and the velocity $v_{y 1}$ of the chain in the vertical direction are:

$$
\begin{aligned}
& v_{x 1}=r_{1} \omega_{1} \cos \alpha \\
& v_{y 1}=r_{1} \omega_{1} \sin \alpha
\end{aligned}
$$

Where $\omega_{1}$ is the angular velocity of the driving wheel, $r_{1}$ is the pitch circle radius of the driving wheel, $\alpha$ is the phase angle of the link hinge on the driving wheel during the meshing process of chain and sprocket ranging from $-\frac{180^{\circ}}{Z_{1}}$ to $\frac{180^{\circ}}{Z_{1}}, Z_{1}$ is the teeth number of the driving wheel.

It can be seen that the chain moves from fast to slowly and from slowly to fast in the direction of the linear velocity when chain rotates one link. And in the vertical direction, the chain moves up and down. With the periodical change of the chain, the chain generates vibration and additional dynamic load. When the chain links are elongated, the radius of the pitch circle elongate. And since the radius of the pitch circle is proportional to the linear velocity of the chain, the linear velocity of the chain also increases.

\section{Step Chain Drive Model Establishment and Simulation Analysis}

\subsection{Dynamic Model Based on SIMPACK.}

SIMPACK is a software specially designed for dynamic analysis of mechanical systems, which has a specific module of chain dynamics, called SIMPACK CHAIN. This paper builds an escalator step chain model. The path that the chain links have to follow is guided using some contact guides. Define the torque on the driving wheel and then define the rotation damping on the driven wheel to add the load, so as to make the step chain wheel rotate. The integration time is set to 8s, output steps is 5000 and the solver is set to LSODE. A marker point is created at the red ellipse marker in figure 2 , and the chain path output result is defined here. Set the chain pitch length to normal state, elongation $1 \%$, elongation $2 \%$ and elongation $3 \%$, and then offline integrate.

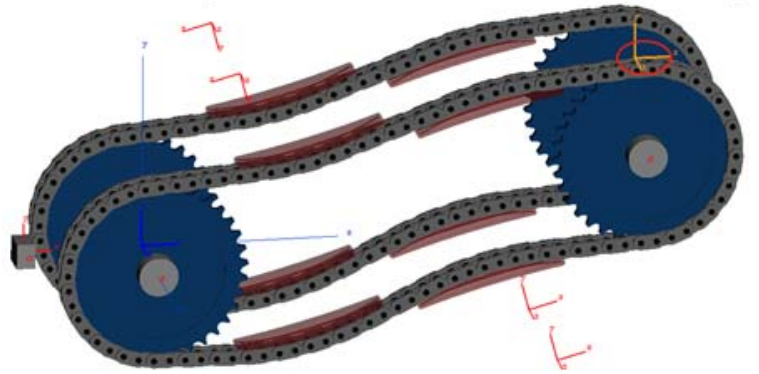

Fig 2. Step chain simulation model

\subsection{Analysis of Simulation Results.}

\subsubsection{Time Domain Analysis.}

Select data after $5 \mathrm{~s}$ as experimental data, when the step chain is running stably. The velocity data of the chain path at normal state and chain pitch elongation state from $5 \mathrm{~s}$ to $8 \mathrm{~s}$ is got, which is shown as figure 3 . 


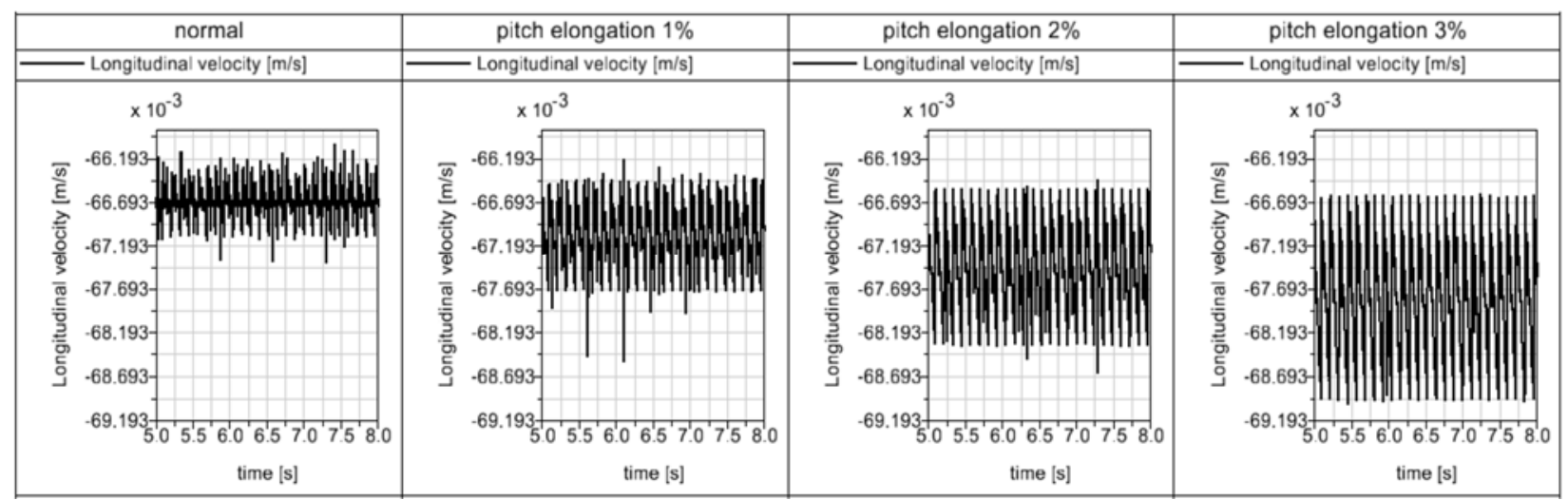

Fig 3. Longitudinal velocity in the chain path

As can be seen from Fig. 3, when the chain pitch is normal and elongated, the time domain waveforms of the velocity signals in chain paths are different. As the pitch of the chain is elongated the range of velocity get wide and the velocity accelerate.

The mean of absolute value, standard deviation, root mean square, kurtosis and waveform factor in different cases are calculated. The calculation results are shown in Table 1 and figure 4.

Table 1 . The time domain indicator of velocity in the chain path

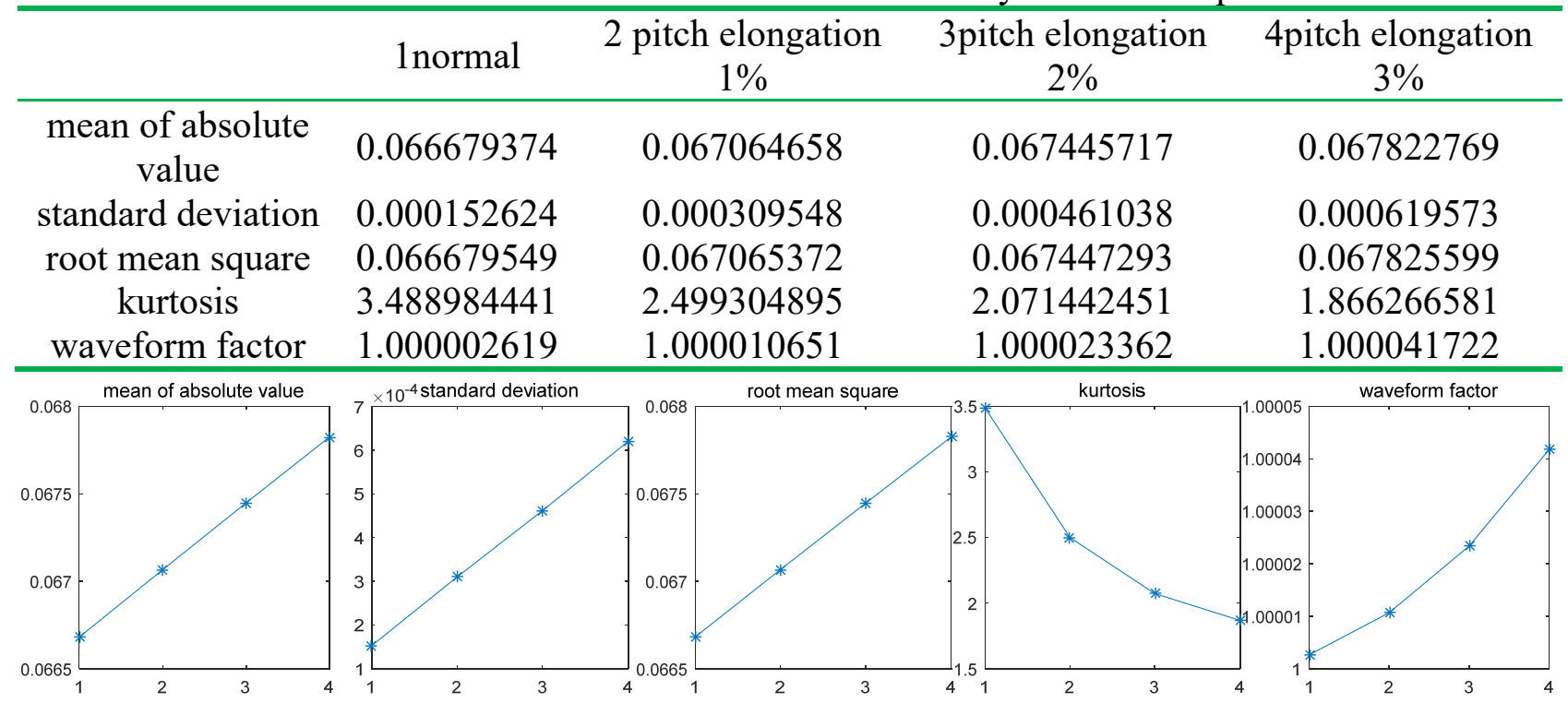

Fig 4. The time domain indicator of velocity in the chain path

From Table 1 and figure 4 we can see that:

(1) When the chain pitch is elongated in different length, the time domain indicator will change significantly.

(2) The time domain indicator means of absolute value, standard deviation, and root mean square increase with the elongation of the chain pitch and the kurtosis decreases with the elongation of the chain pitch. These five indicators can clearly distinguish different chain pitch elongation states.

(3) The means of absolute value, standard deviation, and root mean square show a certain linear relationship in different case.

\subsubsection{Frequency Domain Analysis.}

Fourier transformation is carried out on the chain path velocity signal that has obtained in normal state and chain pitch elongation state. The spectrograms obtained after the transformation are as follows: 

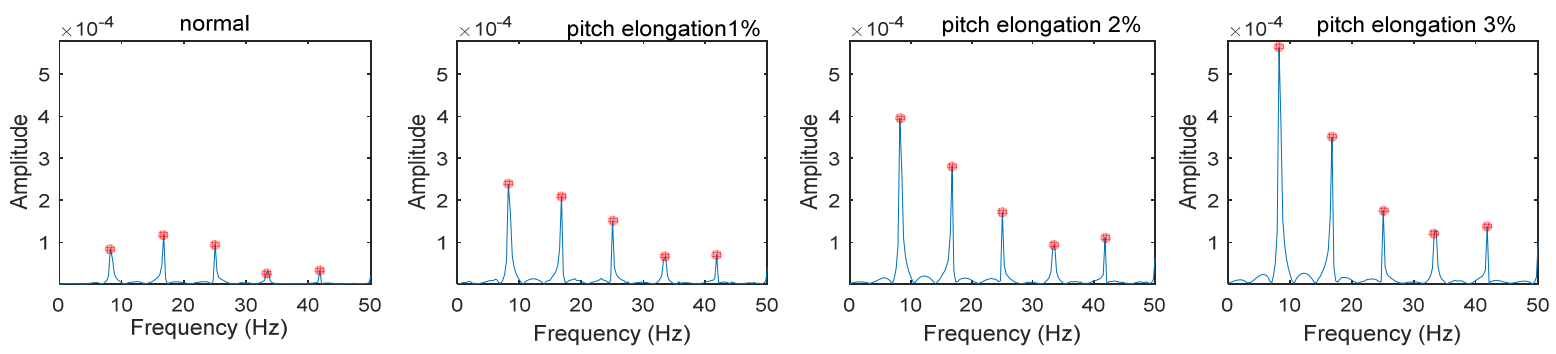

Fig 5. Velocity spectrogram in the chain path

The characteristic frequency $8.242 \mathrm{~Hz}$ is close to the theoretical meshing frequency $8.59 \mathrm{~Hz}$ of the step chain and sprockets. it can be considered as the actual meshing frequency of the step chains.

The red dots in the figure are $f(8.242 \mathrm{~Hz}$, meshing frequency of the step chain $), 2 \mathrm{f}(16.79 \mathrm{~Hz}) 3 \mathrm{f}$ $(25.03 \mathrm{~Hz}), 4 \mathrm{f}(33.27 \mathrm{~Hz})$, and $5 \mathrm{f}(41.82 \mathrm{~Hz})$ respectively. These five frequency multiplications are counted and compared with each other, as shown in Table 2 and figure 6.

Table 2 . The frequency multiplication indicators of chain path velocity

\begin{tabular}{ccccc}
\hline & normal & pitch extension $1 \%$ & pitch extension $2 \%$ & pitch extension $3 \%$ \\
\hline $\mathrm{f}(8.242 \mathrm{~Hz})$ & 0.00008425 & 0.0002375 & 0.0003947 & 0.0005634 \\
$2 \mathrm{f} 16.79 \mathrm{~Hz})$ & 0.0001172 & 0.0002088 & 0.0002811 & 0.0003494 \\
$3 \mathrm{f}(25.03 \mathrm{~Hz})$ & 0.00009495 & 0.0001498 & 0.0001704 & 0.0001752 \\
$4 \mathrm{f}(33.27 \mathrm{~Hz})$ & 0.00002713 & 0.00006571 & 0.00009477 & 0.0001217 \\
$5 \mathrm{f}(41.82 \mathrm{~Hz})$ & 0.00003241 & 0.00007024 & 0.0001109 & 0.0001369 \\
\hline
\end{tabular}

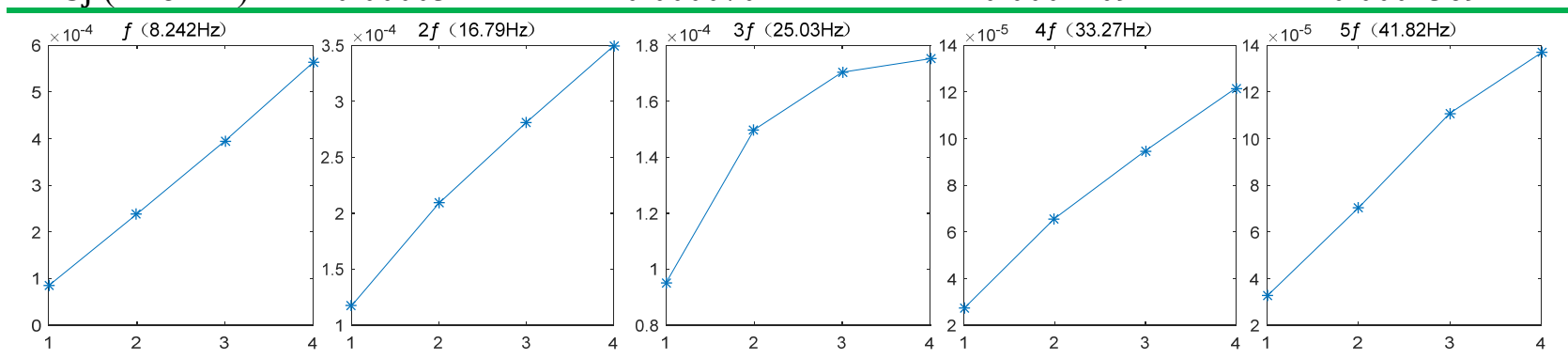

Fig 6 . The frequency multiplication indicators of chain path velocity

From Table 1 and figure 6 we can see that:

(1) When the chain pitch is elongated in different length, the frequency multiplication indicators $f, 2 f, 3 f, 4 f, 5 f$ will change significantly.

(2) These five indicators are sensitive to different elongation of the chain pitch. With the elongation of the chain pitch, these five indicators increase.

\section{Summary}

This paper simulates the motion state of step chain pitch elongated in different length. The simulation results are in accordance with the theoretical calculations, which indicate that both time domain characteristics and frequency domain characteristics of the chain path velocity can reflect the degree of chain pitch elongation. This provides an important basis for the maintenance and maintenance of the step chain.

\section{Acknowledgements}

This work is partly supported by Chinese Nation Key Projece of Research and Development (Contract No. 2017YFB1201203-001).

\section{References}

[1]. Shunshou Lu. Introduce a practical test method of the escalator step roller life. Engineering \& Test, 2003, 43(1):25-25. 
[2]. Meng F Z, Cheng Y B, Li Y N. Fatigue lifetime distribution pattern of automotive chain. Journal of Jilin University, 2006, 36(6):889-892.

[3]. Rong Wang, Chunqiu Guo. Fracture Analysis on Long Axle-Pin of Elevator Chain Lift . Materials for Mechanical Engineering, 2010, 34(12):74-76.

[4]. Chen Peng . Detection and Fault Diagnosis Technology of Scraper Conveyor Chain. CHINA NEW TECHNOLOGIES AND PRODUCTS, 2011(16):159-159.

[5]. Becerra J M C, Moreno J D C, Esteban B S, et al. Methods for Improving Escalators . Iaee, 2008. 